\title{
PSMA-positive Secondary Tumors in 68Ga-PSMA PET/CT Imaging in Patients with Prostate Cancer
}

\author{
Sevda Sağlampınar Karyağar
}

University of Health Sciences Turkey, Prof. Dr. Cemil Taşşığlu City Hospital, Clinic of Nuclear Medicine, İstanbul, Turkey

\section{Abstract}

Objective: The objective of this research was to examine the prostate-specific membrane antigen (PSMA)-positive secondary tumor incidence in 68Ga-PSMA positron emission tomography/computed tomography (PET/CT) imaging in patients with prostate cancer (PCa).

Methods: Data from 605 68Ga-PSMA PET/CT images of 506 PCa patients used for staging or restaging were analyzed retrospectively. Further, documented separately, PSMA-positive lesions were found not to be PCa-related and were then suspected as secondary tumors. The results were analyzed from those lesions that were histopathologically verified.

Results: Nine patients (1.8\%) had a PSMA-positive lesion that was believed to be a secondary tumor. Of these lesions, five (1\%) were histopathologically confirmed, and secondary tumors were diagnosed (namely, squamous cell non-small cell lung cancer, papillary thyroid cancer (TCa) lymph node metastasis, minimally invasive TCa, colon cancer and liver metastases, and fibrohistiocytic tumor). The mean serum PSA value for patients diagnosed with secondary tumor was 29.42 (0.01-142.22) ng/mL. For PSMA-positive secondary tumor lesions, the mean maximum standard unit value was 9.74 (3.9-15).

Conclusion: Especially in the presence of atypical location and insufficient serum PSA values, PSMA-positive lesions should be considered secondary tumors, and differential diagnostic studies should be conducted.

Keywords: ${ }^{88 G a-P S M A ~ P E T / C T, ~ P S M A ~ u p t a k e, ~ s e c o n d a r y ~ t u m o r ~}$

\section{INTRODUCTION}

Prostate-specific membrane antigen (PSMA), also referred to as folate hydrolase I or glutamate carboxypeptidase II, is expressed at elevated levels in prostatic adenocarcinoma prostate cancer (PCa) cells 100-1000 times that of normal prostate tissue $(1,2)$. Moreover, PSMA expression may increase with high tumor grade/ stage and also with tumor dedifferentiation, metastatic disease, and hormone resistance $(1,2)$. This is the target site for $68 \mathrm{Ga}$ labeled agents that bind and cause the agent to be internalized, thereby allowing detection on positron emission tomography (PET) imaging. Compared to traditional imaging, 68Ga-PSMA PET/ computed tomography (CT) has superior diagnostic capabilities to detect tumoral focus in primary staging and biochemical recurrence (BCR) of patients with $\mathrm{PCa}$ (3-5).
68Ga-PSMA uptake can usually be seen in the salivary glands, nasopharynx, vocal cords, thyroid gland, duodenum, small intestines, spleen, liver, pancreas, stomach, adrenal gland, kidneys, rectum, testes, and varying degrees of vertebral bone marrow (6,7). In addition, PSMA expression was demonstrated in a number of nonprostatic malignant and non-malignant conditions found in 68Ga-PSMA PET/CT (8-10) and was also seen in neovascular capillary endothelium in the peritumoral areas of a variety of epithelial malignancies (2). This condition resulted in the discovery of incidental non-prostatic tumors on ${ }^{68 G a-P S M A}$ $\mathrm{PET} / \mathrm{CT}$ imaging performed for both primary staging and BCR and led to its use for diagnostic purposes for malignancies such as hepatocellular Ca and renal $\mathrm{Ca}$. In this study, the incidence of PSMA-positive secondary tumors found in $68 \mathrm{Ga}-\mathrm{PSMA} \mathrm{PET} / \mathrm{CT}$ 
imaging were examined and histopathologic findings of these tumors were evaluated.

\section{METHODS}

\section{Patients}

Retrospectively, the medical history and imaging data of PCa patients who underwent $68 \mathrm{Ga}-\mathrm{PSMA}-\mathrm{PET} / \mathrm{CT}$ at our nuclear medicine department from July 2017 to December 2019 were analyzed. The PSMA-positive lesions believed to be secondary tumors of these patients were examined for imaging and histopathologic findings. Patients with secondary malignancy prior to 68Ga-PSMA-PET/CT imaging were excluded from the study. If lesions were found, localization, CT findings, and clinical findings were evaluated together, and pathologies with no association with PCa and high likelihood of being benign were not included.

All patients signed written informed consent forms for the purpose of reviewing and publishing their results. Hence, this study was accepted by the Ethics Committee of Prof. Dr. Cemil Taşçıoğlu City Hospital (01.07.2020/14).

\section{Imaging and Analysis}

Patients were imaged using an integrated PET/CT scanner consisting of a full-ring HI-REZ LSO PET and a six-slice CT scanner (Siemens Biograph 6, Chicago, IL, USA). Each patient was given a standardized weight-based dose of $2 \mathrm{MBq} / \mathrm{kg}$ (range 70-180 MBq) ${ }^{68}$ Ga-PSMA. Further, PET/CT scan was performed at 60 min post-injection with an emission time of 3 min per bed position from the vertex to the upper thigh. Prior to emission imaging, low-dose CT for attenuation correction and anatomic localization was performed with the following parameters: $50 \mathrm{~mA}, 140$ $\mathrm{kV}$, and $5 \mathrm{~mm}$ section thickness. Image analysis was carried out on the Esoft multimodality computer platform (Siemens Medical Solutions, Erlangen, Germany). The images were then interpreted by an experienced nuclear medicine physician on the basis of a visual examination with knowledge of the patient's clinical history and the findings of previous imaging studies. A positive scan was characterized as such when the PSMA uptake was visually above the background and did not correspond to the physiologic distribution sites. The maximum standardized uptake values $\left(S U V_{\max }\right)$ of each lesion were determined by the region of interest applied in the transaxial attenuation-corrected PET slice with the highest uptake.

Patients were suspected of developing PSMA-positive secondary tumor in the presence of one of the following criteria:

- A lesion with increased PSMA uptake was detected at a site that was unexpected on the basis of the PCa metastasis pattern.

- Inconsistency between PSMA-positive lesion, patient clinical progression, and serum PSA values was found.

Any site of incidental PSMA uptake found not to be PCa-related and suspected of being a secondary tumor was documented separately. PSMA-positive lesions suspected of secondary tumors were verified by histopathologic evaluation whenever possible.

\section{Statistical Analysis}

During the evaluation of the study data, descriptive statistical methods such as mean, median, frequency, ratio, minimum, and maximum value were used.

\section{RESULTS}

Retrospectively, data from 605 68Ga-PSMA PET/CT images performed for staging or restaging of 506 PCa patients were reevaluated. Nine (1.8\%) of these patients were reported as having PSMA-positive lesion suspected of being a secondary tumor. The mean age of patients was 67.4 years (52-79) with six patients having ${ }^{68} \mathrm{Ga}-\mathrm{PSMA}$ PET/CT imaging for restaging and three patients having it for staging purposes. Of these lesions, five (1\%) were histopathologically confirmed and had the following secondary tumor diagnoses (Table 1): squamous cell non-small cell lung cancer (NSCLC) presenting as mass lesion in the left lung (Figure 1), papillary thyroid cancer (TCa) presenting as lymph node in the right cervical region, minimally invasive TCa as a nodular lesion in the right lobe of the thyroid, metastatic colon cancer presenting as multiple hypodense lesions in the liver and lesion in the descending colon, and fibrohistiocytic tumor presenting as subcutaneous

\begin{tabular}{|l|l|l|l|}
\hline Table 1. General characteristics of histopathologically verified PSMA-positive secondary tumor \\
\hline PSMA-positive lesion & PSA & SUV $_{\max }$ & Histopathological diagnosis \\
\hline Mass lesion at left lung & 4.65 & 7.84 & Squamous cell lung cancer \\
\hline Nodular lesion at right thyroid lobe & 0.01 & 15 & Minimally invasive thyroid cancer \\
\hline Lymph node at right cervical chain & 0.01 & 8.09 & Papillary thyroid cancer lymph node metastasis \\
\hline Descending colon lesion to multiple liver lesion & 142.2 & 13.86 & Colon cancer to liver metastases \\
\hline Subcutaneous lesion at dorsal region & 0.2 & 3.9 & Fibrohistiocytic tumor \\
\hline PSMA: Prostate-specific membrane antigen, PSA: Prostate-specific antigen, SUV & max: Maximum standardized uptake values \\
\hline
\end{tabular}


lesion in the dorsal region. Of these patients, the mean serum PSA value was $29.42(0.01-142.22) \mathrm{ng} / \mathrm{mL}$. Cases with PSMApositive secondary tumor diagnosis had a mean $S_{U} V_{\max }$ value of 9.74 (3.9-15). In the case with histopathologic diagnosis of fibrohistiocytic tumor, the SUV ${ }_{\max }$ lesion value was lower than the physiologic SUV max $_{\max }$ values for the liver and higher than the other cases. With histopathologic investigation, three patients with a diagnosis of malignancy were treated, while two cases with biopsy were included in the treatment program for medical oncology. In three patients with increased PSMA expression in the thyroid gland (SUV $V_{\max }$ values 3.54, 5.55, and 2.27), no advanced research could be conducted due to the clinical condition of the patients or follow-up in an outpatient center. A patient with PSMA-positive lesion (SUV $\max 25.13)$ in the spleen parenchyma had a diagnosis of hemangioma with magnetic resonance imaging.

\section{DISCUSSION}

While prostate-specific, PSMA is not specific for PCa. PSMA-positive incidental secondary tumor lesions have been observed since the first application of $68 \mathrm{Ga}$-PSMA PET/CT imaging to PCa patients. PSMA is expressed in various forms of tumor neovasculature, such as renal cell carcinomas, bladder carcinomas, colonic adenocarcinomas, gastric cancers, TCa, gliomas, LC, malignant

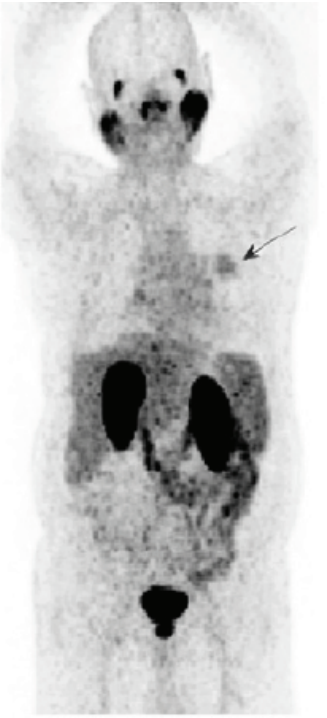

A



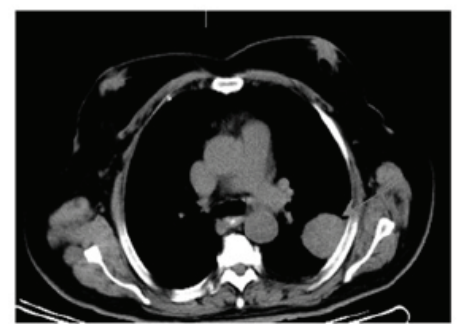

C
Figure 1. A 73-year-old PCa patient is imaged with ${ }^{68} \mathrm{Ga}$ PSMA PET/CT for restaging. The serum PSA value was $4.65 \mathrm{ng} / \mathrm{mL}$. PSMA expressing lung mass was found in the left lung (SUV max $_{\text {: }} 7.84$ ) on ${ }^{68} \mathrm{Ga}$ PSMA PET/ CT imaging (arrow). Histopathological evaluation revealed squamous cell NSCLC

PCa: Prostate cancer, PSMA: Prostate-specific membrane antigen, PET: Positron emission tomography, CT: Computed tomography, SUV $\mathrm{max}_{\max }$ Maximum standardized uptake values, NSCLC: Non-small cell lung cancer melanomas, osteosarcomas, and soft tissue tumors, but not normal tissue vasculature (11-17). There are several publications in the literature on incidental secondary malignancies found on ${ }^{68} \mathrm{Ga}-\mathrm{PSMA} \mathrm{PET} / \mathrm{CT}$ imaging performed for the purpose of staging or restaging patients with PCa $(18,19)$. Hepatocellular carcinoma, renal adenocarcinoma, follicular and papillary TCa, follicular lymphoma, multiple myeloma, gastrointestinal stromal tumor, rectal adenocarcinoma, colon adenocarcinoma, penile squamous cell carcinoma (SCC), primary LC, breast cancer, urothelial cancer, and oropharynx SCC (20-33) were included in these articles.

In this 506-patient cohort group, $1.8 \%$ of patients were reported as having PSMA-positive incidental lesions which were suspected as a secondary tumor. of these lesions, five (1\%) were histopathologically verified, and secondary tumor diagnosis was made (papillary TCa, minimally invasive TCa, NSCLC, colon cancer, and fibrohistiocytic tumor). Osman et al. (34) observed synchronous primary malignancy in 5 patients in a 764 PCa case series of PET/CT imaging performed (0.7\%, 2 lung adenocarcinoma, 1 diffuse B-cell lymphoma, 1 PCa, and 1 SCC of the base of the tongue).

In this study, TCa was the most frequently identified secondary incidental tumor. A diagnosis of metastatic papillary TCa was made in one patient with histopathologic examination of lymph nodes in the cervical region. This patient had a papillary TCa focus of $0.5 \mathrm{~cm}$ identified with thyroidectomy pathology. Pathologic involvement of the thyroid gland was not present on 68Ga-PSMA PET/CT imaging. It was concluded that the small size of the primary lesion was effective due to the lack of detection of PSMA-positive lesion in the thyroid gland. One patient had minimally invasive follicular TCa diagnostic sites with thyroidectomy pathology of PSMA-positive thyroid lesion. Immunohistochemistry studies of TCa cases showed that neovascular PSMA expression was more common in TCa relative to benign thyroid pathologies and that there were high levels of PSMA expression in poorly or undifferentiated and aggressive TCa $(13,34)$. This property resulted in the use of 68Ga-PSMA PET/ $\mathrm{CT}$ imaging and potential radionuclide treatment for metastasis identification in high thyroglobulin and radioiodine-negative TCa, in particular (35).

One of our cases diagnosed with an incidental PSMA-positive secondary tumor had squamous cell NSCLC. In the literature, both squamous and adenocarcinoma NSCLC cases have been reported to be identified with 68Ga-PSMA PET/CT imaging $(28,36,37)$. Schmidt et al. (11) immunohistochemistry studies on NSLC cases found neovascular PSMA expression in 49\% of NSCLC 
and tumor cell PSMA expression in 6\%. High neovascular PSMA expression was also reported to be associated with higher tumor grading.

In this study, a patient with PSMA-positive colon cancer in the descending colon also demonstrated PSMA expression in liver metastases. Cases of PSMA-positive colorectal cancer have been documented in the literature, and immunohistochemistry studies have reported that higher-grade colorectal tumors appear to have higher PSMA expression and higher risk of distant metastases and vascular invasion. However, there was no statistical difference in overall survival or disease-free survival based on PSMA expression $(16,27,28)$.

In analysis, the mean serum PSA value for five patients with histopathologic verification of secondary tumor diagnosis was $29.42(0.01-142.22) \mathrm{ng} / \mathrm{mL}$. There were two patients with $0.01 \mathrm{ng} / \mathrm{mL}$ and one patient with $0.2 \mathrm{ng} / \mathrm{mL}$, whereas three patients had low serum PSA value with PSMA-positive lesion identified. When lesions are evaluated with anatomic localization, the low serum PSA value should be assessed as a factor warning of the presence of pathology outside the prostate.

\section{Study Limitations}

The most significant limitation of this retrospectively designed study is that all incidental PSMA-positive lesions found on ${ }^{68} \mathrm{Ga}$ PSMA PET/CT imaging were not histopathologically verified. Another drawback is that differential diagnosis of synchronous secondary malignancies could not be made because advanced diagnostic studies could not be conducted in patients with extensive visceral metastases due to the clinical status and ethical reasons.

\section{CONCLUSION}

PCa patients may rarely have PSMA-positive secondary tumors found in ${ }^{8} \mathrm{Ga}$ PSMA PET/CT images. In particular, considering the CT properties of the atypical PCa metastasis location and the presence of PSMA-positive lesions that do not respond to patient clinical symptoms, consideration should be given to the possibility of secondary tumors and differential diagnostic studies be performed.

\section{Ethics}

Ethics Committee Approval: Ethics Committee of Prof. Dr. Cemil Taşçıŏlu City Hospital (01.07.2020/14).

Informed Consent: All patients signed written informed consent forms for the purpose of reviewing and publishing their results.
Peer-review: Externally peer-reviewed.

Financial Disclosure: The author declared that this study received no financial support.

\section{REFERENCES}

1. Perner S, Hofer MD, Kim R, Shah RB, Li H, Möller P, et al. Prostatespecific membrane antigen expression as a predictor of prostate cancer progression. Hum Pathol 2007;38:696-701.

2. Silver DA, Pellicer I, Fair WR, Heston WD, Cordon-Cardo C. Prostatespecific membrane antigen expression in normal and malignant human tissues. Clin Cancer Res 1997;3:81-5.

3. Yaxley JW, Raveenthiran S, Nouhaud FX, Samaratunga H, Yaxley WJ, Coughlin G, et al. Risk of metastatic disease on 68gallium-prostatespecific membrane antigen positron emission tomography/computed tomography scan for primary staging of 1253 men at the diagnosis of prostate cancer. BJU Int 2019;124:401-7.

4. Caroli P, Sandler I, Matteucci F, De Giorgi U, Uccelli L, Celli M, et al. 68Ga-PSMA PET/CT in patients with recurrent prostate cancer after radical treatment: prospective results in 314 patients. Eur J Nucl Med Mol Imaging 2018;45:2035-44.

5. Karyagar SS, Dincer ST. The diagnostic power of the 68Ga PSMA PET-CT in biochemical recurrence after primary curative treatment in patients with prostate cancer: A single center experience. Eur Arch Med Res 2019;35:241-4

6. Özülker F. Assessment of physiological distribution and normal variants of 68Ga PSMA-I\&T PET/CT. Eur Arch Med Res 2018;34:235-42.

7. Demirci E, Sahin OE, Ocak M, Akovali B, Nematyazar J, Kabasakal L. Normal distribution pattern and physiological variants of 68GaPSMA-11 PET/CT imaging. Nucl Med Commun 2016;37:1169-79.

8. Pyka T, Weirich G, Einspieler I, Maurer T, Theisen J, Hatzichristodoulou G, et al. 68Ga PSMA HBED CC PET for differential diagnosis of suggestive lung lesions in patients with prostate cancer. J Nucl Med 2016;57:36771.

9. Sasikumar A, Joy A, Nanabala R, Pillai MR, Thomas B, Vikraman KR, et al. 68Ga PSMA PET/CT imaging in primary hepatocellular carcinoma. Eur J Nucl Med Mol Imaging 2016;43:795-6.

10. Sawicki LM, Buchbender C, Boos J, Giessing M, Ermert J, Antke C, et al. Diagnostic potential of PET/CT using a 68Ga labelled prostate specific membrane antigen ligand in whole body staging of renal cell carcinoma: Initial experience. Eur J Nucl Med Mol Imaging 2017;44:1027.

11. Schmidt LH, Heitkötter B, Schulze AB, Schliemann C, Steinestel K, Trautmann $M$, et al. Prostate specific membrane antigen (PSMA) expression in non-small cell lung cancer. PLoS One 2017;12:e0186280.

12. Ghosh A, Heston WD. Tumor target prostate specific membrane antigen (PSMA) and its regulation in prostate cancer. J Cell Biochem 2004;91:52839.

13. Heitkötter B, Steinestel K, Trautmann M, Grünewald I, Barth P, Gevensleben $\mathrm{H}$, et al. Neovascular PSMA expression is a common feature in malignant neoplasms of the thyroid. Oncotarget 2018;9:9867-74.

14. Zeng C, Ke ZF, Yang Z, Wang Z, Yang SC, Luo CQ, et al. Prostate-specific membrane antigen: a new potential prognostic marker of osteosarcoma. Med Oncol 2012;29:2234-9.

15. Heitkotter B, Trautmann M, Grunewald I, Bogemann M, Rahbar K, Gevensleben $\mathrm{H}$, et al. Expression of PSMA in tumor neovasculature of 
high grade sarcomas including synovial sarcoma, rhabdomyosarcoma, undifferentiated sarcoma and MPNST. Oncotarget 2017;8:4268-76.

16. Haffner MC, Kronberger IE, Ross JS, Sheehan CE, Zitt M, MühImann G, et al. Prostate-specific membrane antigen expression in the neovasculature of gastric and colorectal cancers. Hum Pathol 2009;40:1754-61.

17. Bychkov A, Vutrapongwatana U, Tepmongkol S, Keelawat S. PSMA expression by microvasculature of thyroid tumors - Potential implications for PSMA theranostics. Sci Rep 2017;7:5202.

18. Malik D, Kumar R, Mittal BR, Singh H, Bhattacharya A, Singh SK. 68Ga Labeled PSMA Uptake in Nonprostatic Malignancies: Has the Time Come to Remove "PS" From PSMA? Clin Nucl Med 2018:43:529-32.

19. Sheikhbahaei S, Afshar-Oromieh A, Eiber M, Solnes LB, Javadi MS, Ross AE. Pearls and pitfalls in clinical interpretation of prostate-specific membrane antigen (PSMA)-targeted PET imaging. Eur J Nucl Med Mol Imaging 2017; 44:2117-36.

20. Shetty D, Patel D, Le K, Bui C, Mansberg R. Pitfalls in Gallium-68 PSMA PET/CT interpretation-a pictoral review. Tomography 2018;4:182-93.

21. Sasikumar A, Joy A, Nanabala R, Unni M, Tk P. Complimentary pattern of uptake in 18F-FDG PET/CT and 68Ga-prostate-specific membrane antigen PET/CT in a case of metastatic clear cell renal carcinoma. Clin Nucl Med 2016;41:517-9.

22. Sager S, Vatankulu B, Uslu L, Sonmezoglu K. Incidental detection of follicular thyroid carcinoma in 68Ga-PSMA PET/CT imaging. J Nucl Med Technol 2016;44:199-200.

23. Jena A, Zaidi S, Kashyap V, Jha A, Taneja S. PSMA expression in multinodular thyroid neoplasm on simultaneous 68Ga-PSMA PET/MRI. Indian J Nucl Med 2017;32:159-61.

24. Kanthan GL, Coyle L, Kneebone A, Schembri GP, Hsiao E. Follicular lymphoma showing avid uptake on 68Ga PSMA HBED-CC PET/CT. Clin Nucl Med 2016;41:500-1.

25. Sasikumar A, Joy A, Pillai MR, Nanabala R, Thomas B. 68Ga PSMA PET/CT Imaging in Multiple Myeloma. Clin Nucl Med 2017;42:126-7.

26. Noto B, Weckesser M, Buerke B, Pixberg M, Avramovic N. Gastrointestinal Stromal tumor showing intense tracer uptake on PSMA PET/CT. Clin Nucl Med 2017;42:200-2.

27. Huang YT, Fong W, Thomas P. Rectal carcinoma on 68Ga-PSMA PET/CT. Clin Nucl Med 2016;41:167-8.
28. Hangaard L, Jochumsen MR, Vendelbo MH, Bouchelouche K. Metastases from colorectal cancer avid on 68Ga-PSMA PET/CT. Clin Nucl Med 2017;42:532-3.

29. Froehner M, Kuithan F, Zophel K, Heberling U, Laniado M, Wirth MP. Prostate-specific membrane antigen-targeted Ligand positron emission tomography/computed tomography and Immunohistochemical findings in a patient with synchronous metastatic penile and prostate cancer. Urology 2017;101:5-6.

30. Shetty D, Loh H, Bui C, Mansberg R, Stevanovic A. Elevated 68Ga prostate-specific membrane antigen activity in metastatic non-small cell lung cancer. Clin Nucl Med 2016;41:414-6.

31. Sathekge M, Modiselle M, Vorster M, Mokgoro N, Nyakale N, Mokaleng B, et al. 68Ga-PSMA imaging of metastatic breast cancer. Eur J Nucl Med Mol Imaging 2015;42:1482-3.

32. Gupta M, Choudhury PS, Gupta G, Gandhi J. Metastasis in urothelial carcinoma mimicking prostate cancer metastasis in 68Ga- prostatespecific membrane antigen positron emission tomography-computed tomography in a case of synchronous malignancy. Indian J Nucl Med 2016;31:222-4.

33. Lawhn-Heath C, Flavell RR, Glastonbury C, Hope TA, Behr SC. Incidental detection of head and neck Squamous cell carcinoma on 68Ga-PSMA-11 PET/CT. Clin Nucl Med 2017;42:218-20.

34. Osman MM, Iravani A, Hicks RJ, Hofman MS. Detection of Synchronous Primary Malignancies with 68Ga-Labeled Prostate-Specific Membrane Antigen PET/CT in Patients with Prostate Cancer: Frequency in 764 Patients. J Nucl Med 2017;58:1938-42.

35. Sollini M, di Tommaso L, Kirienko M, Piombo C, Erreni M, Lania AG, et al. PSMA expression level predicts differentiated thyroid cancer aggressiveness and patient outcome. EJNMMI Res 2019;9:93.

36. Sasikumar A, Joy A, Pillai MRA, Oommen KE, Jayakumar R. Rare Case of Intratracheal Metastasis Detected on 68Ga-Prostate-Specific Membrane Antigen PET/CT Scan in a Case of Thyroglobulin Elevated Negative Iodine Scan Syndrome. Clin Nucl Med 2018;43:282-3.

37. Pyka T, Weirich G, Einspieler I, Maurer T, Theisen J, Hatzichristodoulou G. 68Ga-PSMA-HBED-CC PET for Differential Diagnosis of Suggestive Lung Lesions in Patients with Prostate Cancer. J Nucl Med 2016:57:36771. 